\title{
Case Report on Intracranial Hemorrhage Related to Type I Cryoglobulinemia
}

\author{
Richard Dalyai, MD, L. Fernando Gonzalez, MD \\ Department of Neurological Surgery, Thomas Jefferson University, Philadelphia, PA
}

\section{Introduction}

Atraumatic intracranial hemorrhage (ICH) is a common neurosurgical pathology that affects about 20 of 100,000 people every year with a mortality of at least $40 \%{ }^{1}$ However, a specific etiology cannot be identified in about $30 \%$ of spontaneous ICH. ${ }^{1}$ Here, we present a rare case of ICH related to a patient's previously undiagnosed cryoglobinemia.

Cryoglobulinemia is a chronic systemic disease characterized by the presence of immunoglobulins that reversibly precipitate at low temperatures (approximately $37 \mathrm{deg} \mathrm{C}$ ) and re-dissolve at higher temperatures. ${ }^{2}$ The cryoglobulins and resulting cryoprecipitate disturbs the normal hematologic parameters though three pathways: vasculitis through immune complex deposition, hyper-viscosity from protein obstruction, and abnormal platelet aggregation. ${ }^{2}$ Microscopic study of cryoglobulin tissue reveals leukoclastic vasculitis and hyaline thrombus which predominantly leads to thrombosis. ${ }^{2}$ With these mechanisms in mind, one can understand how cryoglobulinema typically presents as a pro-thrombotic disorder, with purpura, arthralgias, weakness, renal and liver involvement, and peripheral neuropathy. It is not as clear, however, how this disease process can predispose one to bleeding. This was a unique case where the patient presented with coinciding manifestations of clotting in peripheral extremities and intracranial bleeding. Furthermore, both complications of cryoglobinemia were successfully treated.

\section{Case Report}

A 59 year-old gentleman with a known past medical history of intravenous drug abuse, Hepatitis B, Hepatitis C, and HIV presented at an outside hospital with necrotic digits and lethargy. Prior to treatment with a continuous infusion of heparin, a CT scan of the head was performed to rule out an intracranial hemorrhage due to the patient's abnormal mental status and lethargy. This imaging

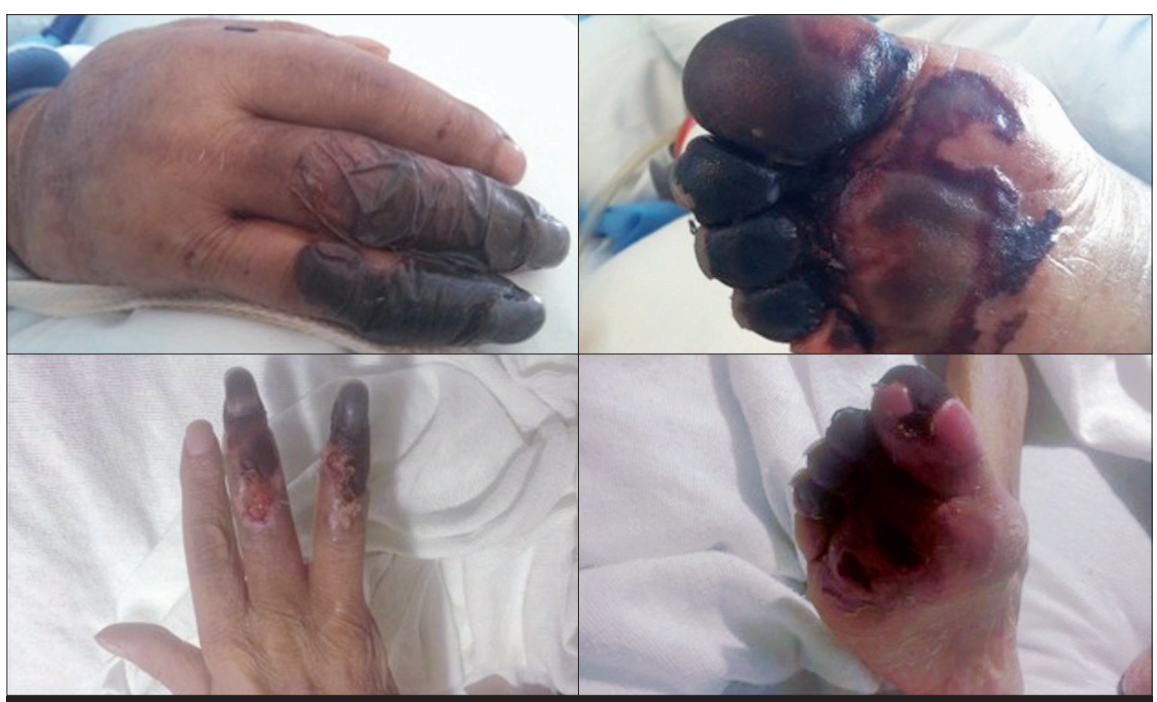

Figure 1

Digit necrosis at admission (A and $B$ ) and towards the end of inpatient course (C and D) study revealed a large right frontal intraparenchymal hemorrhage. The patient was intubated at the outside institution because of concern for neurologic deterioration.

On admission to our institution the patient was opening his eyes to verbal prompting and following commands in all four extremities symmetrically with $3 / 5$ strength; his neurologic exam was otherwise non-focal. In addition, the patient demonstrated necrotic digits in all four extremities, which was worse in his right upper and lower extremity (Figure 1A and 1B). A repeated CT of the patient's head at our facility demonstrated a right frontal intraparenchymal hematoma measuring $6 \times 4 \times 5 \mathrm{~cm}$ or approximately $120 \mathrm{cc}$ in volume (Figure $2 \mathrm{~A}$ ). To further evaluate the hemorrhage for an underlying etiology, a brain MRI with and without gadolinium and head MRA was performed which failed to reveal any underlying mass, aneurysm, or vascular malformation. Given the high level of suspicion for an underlying vascular malformation as the etiology for the hemorrhage, a CT angiogram of the head was also performed, which was also negative for any underlying vascular source. On arrival, complicating treatment of the patient's ICH his INR measured at 1.68 and was corrected to 1.22 with administration of a combination of prothrombin complex factor IX concentrate, FFP, and Vitamin K. The Hematology service was consulted to assist with management of the patient's hepatic coagulopathy. After hematologic consultation, multiple hematologic panels were drawn to help elucidate the etiology of the patient's underlying coagulation disorder - ultimately revealing an IgM of $90 \%$, consistent with cryoglobulinemia.

On hospital day 2, the patient became hemiparetic on his left side and stopped following commands. A repeat CT head demonstrated a stable large frontal hemorrhage. At this time, the patient's poor prognosis was discussed with the patient's family, who desired aggressive and immediate surgical intervention. The patient initially did well post-operatively after his right frontal craniotomy (Figure 2B) and evacuation of the hematoma; until post-operative day 12 when he again developed a left-sided hemiparesis and confusion. A repeat head CT then 


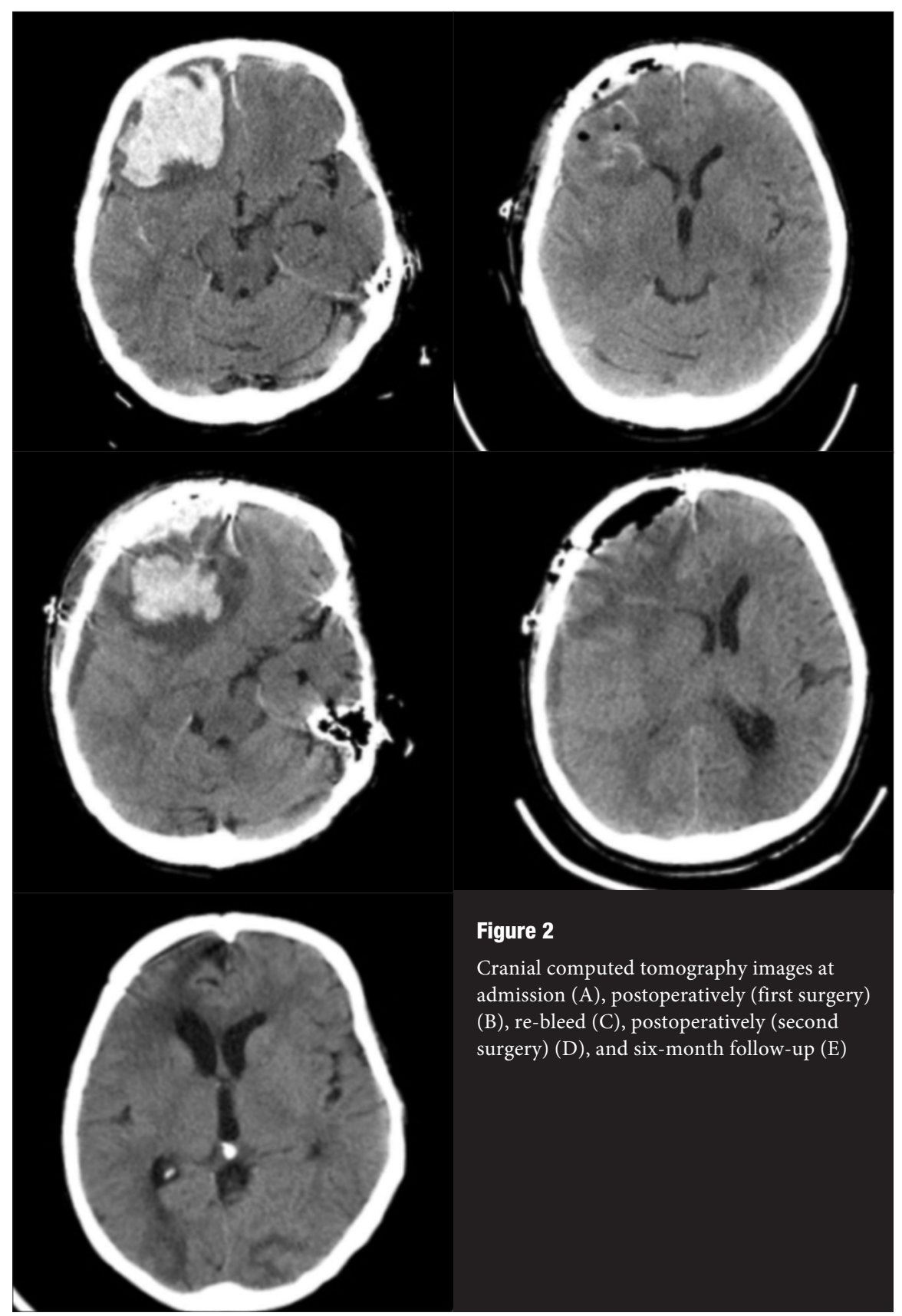

revealed a re-accumulation of his right frontal hematoma and midline shift of $15 \mathrm{~mm}$ (Figure 2C). Once again, after discussions were held with the patient's family regarding their wishes, the patient was taken back to the operating room for repeat right frontal craniotomy and re-evacuation of his recurrent hematoma. Once again, the patient did well post-operatively and returned to his preoperative neurologic exam and successful evacuation apparent on head CT (Figure 2D). During his hospital stay, he was also removed prior to his discharge. The patient's digit necrosis also improved during hospitalization (Figure 1C and 1D). At 6 month follow-up the patient was neurologically intact with a stable CT of his head (Figure 2E).

\section{Discussion}

Cryoglobinemia presenting with neurologic sequelae is rare with few published case reports. ${ }^{3,4}$ In our unusual case, cryoglobulinemia manifested as both hemorrhagic and ischemic changes in two different organ systems. This case illustrates the importance of thoroughly investigating the etiology of an intracerebral hemorrhage with both imaging and lab studies as it allowed for the appropriate multidisciplinary treatments for the patient.

As only a few case reports exist where patients with cryoglobulinemia present with a hemorrhage, the pathophysiology of this feature is not well understood. One hypothesis is that the vasculitis involved in this disease process leaves intracranial vessels more friable and therefore susceptible to rupture. ${ }^{3,4}$ In addition, increased platelet aggregation and hyaline thrombus formation may lead to a situation where focal intracranial blood pressure increases as blood flow is halted by a thrombosed vessel. ${ }^{3,4}$ With these two factors combined, increased blood pressure exerted on a weakened vascular wall may predispose these patients to hemorrhage. Nevertheless, it is not entirely clear why this occurred intra-cranially and why other foci of hemorrhage have not been described. Unfortunately, microscopic examination of tissue removed during the initial evacuation of the intracranial hematoma only revealed organizing hematoma and provided no clear clues to whether or not the patient demonstrated signs of cryoglobulinemia in his intracranial vessels.

\section{Conclusion}

We present a case of cryoglobulinemia related ICH where understanding the background of the patient's bleeding diathesis and thrombotic disorder enabled successful surgical intervention and medical treatment of a large intra-cerebral hematoma.

\section{References:}

ment of his newly-diagnosed cryoglobulinemia. After a prolonged hospital course complicated by pneumonia, ventilation-dependent respiratory failure, and Escherichia coli bacteremia, the patient was eventually discharged to an inpatient rehabilitation facility. At the time of discharge, he was alert and oriented to person, place, and time, following commands in all four extremities with $4 / 5$ strength. He was ambulatory and his tracheostomy tube

1. M Dennis, Outcome after brain haemorrhage, Cerebrovas Dis 16 (2003) (suppl 1), pp. 9-13.

2. E Morra. Cryoglobulinemia. American Society of Hematology. 2005 pp.368-372.

3. L. Mazzola, et. al. Brain hemorrhage as a complication of type I cryoglobulinemia vasculopathy. J Neurol. (2003) 250:1376-1378.

4. W. Chen, et al. Type II Cryoglobulinemia and Brain Hemorrhage. Clinical and Applied Thrombosis/ Hemostasis (2008) Vol 14.2 pp 241-244. 